Original contribution

\title{
Autocalibrated parallel imaging reconstruction with sampling pattern optimization for GRASE: APIR4GRASE
}

\author{
Chaoping Zhang ${ }^{\mathrm{a}, \mathrm{b}, *}$, Alexandra Cristobal-Huerta ${ }^{\mathrm{b}}$, Juan A. Hernandez-Tamames ${ }^{\mathrm{b}}$, \\ Stefan Klein ${ }^{\mathrm{a}, \mathrm{b}}$, Dirk H.J. Poot ${ }^{\mathrm{a}, \mathrm{b}}$ \\ a Department of Medical Informatics, Erasmus MC, Rotterdam, the Netherlands \\ ${ }^{\mathrm{b}}$ Department of Radiology and Nuclear Medicine, Erasmus MC, Rotterdam, the Netherlands
}

\section{A R T I C L E I N F O}

\section{Keywords:}

Magnetic resonance imaging

Reconstruction

GRASE

Parallel imaging

Virtual coil

\begin{abstract}
A B S T R A C T
Purpose: To reduce artifacts and scan time of GRASE imaging by selecting an optimal sampling pattern and jointly reconstructing gradient echo and spin echo images.

Methods: We jointly reconstruct images for the different echo types by considering these as additional virtual coil channels in the novel Autocalibrated Parallel Imaging Reconstruction with Sampling Pattern Optimization for GRASE (APIR4GRASE) method. Besides image reconstruction, we identify optimal sampling patterns for the acquisition. The selected optimal patterns were validated on phantom and in-vivo acquisitions. Comparison to the conventional GRASE without acceleration, and to the GRAPPA reconstruction with a single echo type was also performed.

Results: Using identified optimal sampling patterns, APIR4GRASE eliminated modulation artifacts in both phantom and in-vivo experiments; mean square error (MSE) was reduced by $78 \%$ and $94 \%$, respectively, compared to the conventional GRASE with similar scan time. Both artifacts and g-factor were reduced compared to the GRAPPA reconstruction with a single echo type.

Conclusion: APIR4GRASE substantially improves the speed and quality of GRASE imaging over the state-of-theart, and is able to reconstruct both spin echo and gradient echo images.
\end{abstract}

\section{Introduction}

Reduction of MRI acquisition time is important for increasing patient comfort and lowering scanning costs. To this end, fast imaging techniques have been developed such as Rapid Acquisition with Relaxation Enhancement (RARE), also known as Turbo-Spin-Echo (TSE) or Fast-Spin-Echo (FSE). They acquire multiple spin echoes (SE) during each repetition time (TR) [1]. However, Specific Absorption Rate (SAR) constraints at high magnetic field (3T or higher) limit the speed of FSE due to the large number of refocusing pulses played along each echo train (ET).

The Gradient- and Spin-Echo (GRASE) imaging method can alleviate the SAR constraints of FSE, by performing an Echo-planar imaging (EPI) [2] sampling of gradient echoes (GRE) around each SE [3] between radiofrequency (RF) refocusing pulses. This can also reduce scanning time because gradient polarity switching of EPI requires less time than a RF refocusing pulse with surrounding crusher gradients. However, $T_{2}^{*}$ decay and off-resonance effects cause phase and amplitude modulation, respectively, between SE and GRE, as illustrated in Fig. 1. Both can result in blurring and artifacts, depending on the time ordering of echoes over the k-space phase encoding (PE) positions, called trajectory. Some trajectories, such as vGRASE [4], PROPELLER [5] and cylindrical [6], were proposed to alleviate artifacts for specific applications. In general, for 3D-GRASE imaging, trajectories clustering SE and GRE in k-space separately reduce artifacts to some extent. Specifically, the conventional GRASE with the SORT phase encoding strategy [7] (SORT GRASE), including its extensions to parallel imaging and proton density or $T_{1}$ weighted imaging [8], clusters $\mathrm{SE}$ in the center and GRE in the edges of k-space, producing SE-dominant contrast. Nevertheless, some signal modulation can remain $[7,8]$. Moreover, the large PE steps between SE and GRE in a SORT GRASE acquisition may introduce eddy currents, introducing additional artifacts $[9,10]$.

To correct amplitude and phase modulation between echoes, the independent phase reconstruction of single-slab 3D variable flip angle (VFA) GRASE method has been proposed [11,12]. This method fills the full k-space with interleaved SE and GRE measurements, so that the

\footnotetext{
*Corresponding author at: Departments of Medical Informatics and Radiology, Erasmus Medical Center, 's-Gravendijkwal 230, Rotterdam 3015CE, the Netherlands.

E-mail address: c.zhang@erasmusmc.nl (C. Zhang).
} 


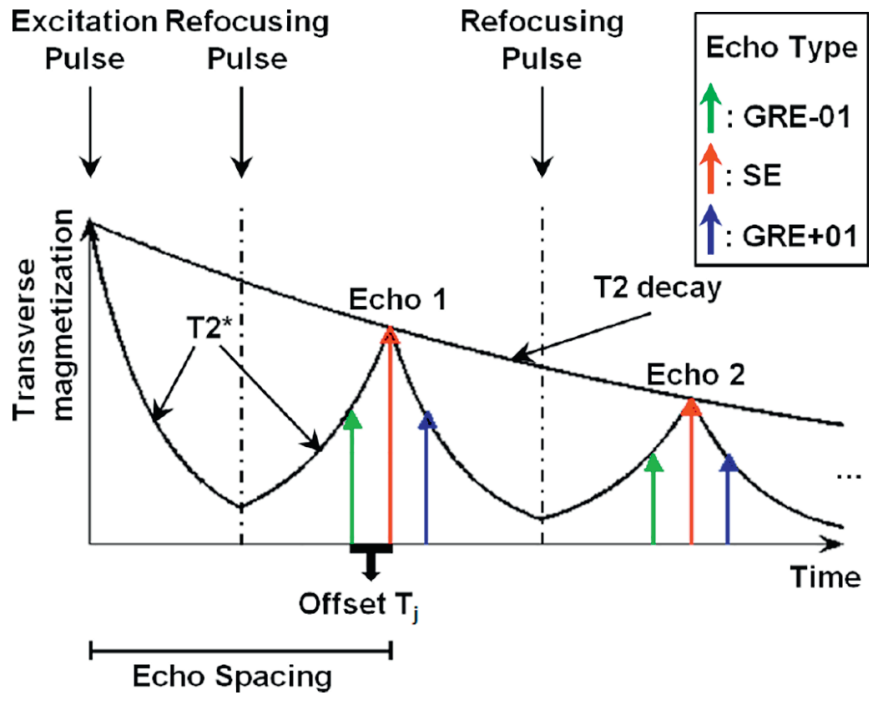

Fig. 1. Signal evolution during a FSE acquisition, showing contributions from $T_{2}$ and $T_{2}^{*}$ decay. The time offset between GRE and SE within one refocusing period witnesses an amplitude modulation by $T_{2}^{*}$ decay and a phase modulation by off-resonance in GRASE acquisition.

different echoes provide complementary spatially information. Subsequently, it compensates the phase differences between echo types and reconstructs the image without modulation artifacts. However, in $[11,12]$ the acceleration capability being evaluated is only the EPI factor, as in the entire acquisition all k-space positions are acquired.

Parallel imaging is able to accelerate scanning by regularly subsampling k-space and using a multi-channel (parallel) coil set in which the coil channels differ in spatial sensitivity. The sensitivity maps can be either used to unwrap the aliasing of the reconstructed image from the subsampled k-space (SENSE [13]) or to predict the unsampled data in the k-space (GRAPPA [14] or ARC [15]). Recently, JVC-GRAPPA [16] was proposed to treat all echoes in FSE-like sequences, or phase cycles in balanced stead-state free precession, along one ET as virtual coils, and jointly reconstruct the image for each of them with a unique contrast.

Building further on the ideas presented in $[11,12,16]$, we propose a

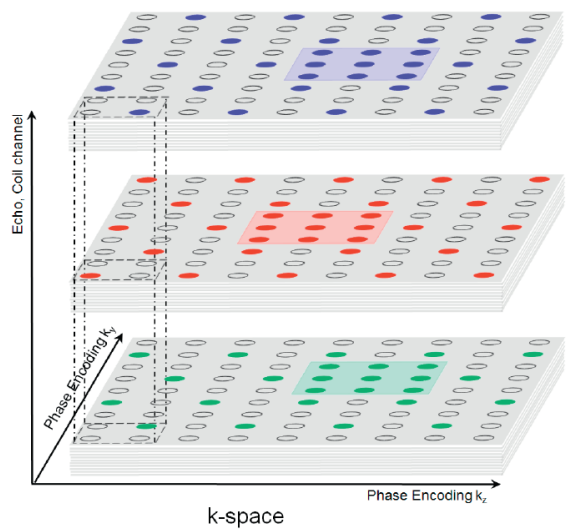

(a) An APIR4GRASE k-space pattern example, regarding echo types as virtual coil channels. rapid GRASE imaging technique that acquires sparse SE and GRE measurements according to an optimized sampling pattern, and subsequently uses autocalibrated parallel imaging reconstruction (APIR) to reconstruct the full k-space from these highly subsampled SE and GRE k-spaces. In this method, henceforth called "APIR4GRASE", the different echo types (SE and GRE) are included as virtual coil channels, but, like normal FSE, the echoes along the echo train are distributed in a single k-space. Optimal k-space patterns are established retrospectively through an exhaustive search, and validated prospectively. By exploiting both SE and GRE measurements and using these optimized subsampling patterns, we obtain a highly accelerated MRI protocol.

\section{Materials and methods}

\section{1. $k$-Space sampling pattern}

APIR4GRASE aims to compensate differences in amplitude and phase among echo types by treating these as virtual coil channels. First, for the SE, a uniformly subsampled k-space grid with echo ordering similar to FSE trajectories is constructed. Next, the sampling grid of GRE is obtained by shifting the SE grid by $\boldsymbol{v} \in \mathbb{Z}^{2}$ in the PE directions. Similar to conventional parallel imaging techniques, the center of each $\mathrm{k}$-space is fully sampled as the auto-calibration signal (ACS) region.

Specifically, the sampled PE positions in SE k-space of APIR4GRASE are defined as $\boldsymbol{k}_{S E}$ :

$$
\begin{aligned}
\boldsymbol{k}_{S E}= & \left\{\left(\left[\begin{array}{ll}
s_{1} & s h r_{1} \\
s h r_{2} & s_{2}
\end{array}\right]\left[\begin{array}{l}
m \\
n
\end{array}\right]\right)^{T} \forall m, n \in \mathbb{Z}\right\} \\
& \cup\left\{[m, n] ;|m| \leqslant A C S_{1},|n| \leqslant A C S_{2}\right\},
\end{aligned}
$$

where $s=\left[s_{1}, s_{2}\right]$ with $s_{i} \in \mathbb{Z}^{+}$contains the subsampling factors in two $\mathrm{PE}$ directions, and $s h r=\left[s h r_{1}, s h r_{2}\right] \in \mathbb{N}^{2}$ is the grid shearing, which is similar to CAIPIRINHA [17]. $A C S_{1}$ and $A C S_{2}$ define the size of the ACS region in the PE1 and PE2 directions. The number of echoes between successive refocusing pulses is defined as the EPI factor $E$. The sampled positions of GRE are shifted relative to the SE positions as $\boldsymbol{k}_{G R E j}=\boldsymbol{k}_{S E}+\boldsymbol{v}_{j},|j| \leqslant\lfloor E / 2\rfloor, j \neq 0, j \in \mathbb{Z}$.

Fig. 2 shows one example of a sampling pattern for APIR4GRASE with $E=3$ with echo types $\mathrm{GRE}_{-1}, \mathrm{SE}$ and $\mathrm{GRE}_{+1} ; k_{y}$ and $k_{z}$ are in PE1 and PE2 directions respectively; $\boldsymbol{s}=[2,2], \boldsymbol{s h} \boldsymbol{r}=[0,1], \boldsymbol{v}_{-1}=[1,1]$, $\boldsymbol{v}_{+1}=[0,1]$ and $A C S_{1}=A C S_{2}=1$. Note that the position of the ACS

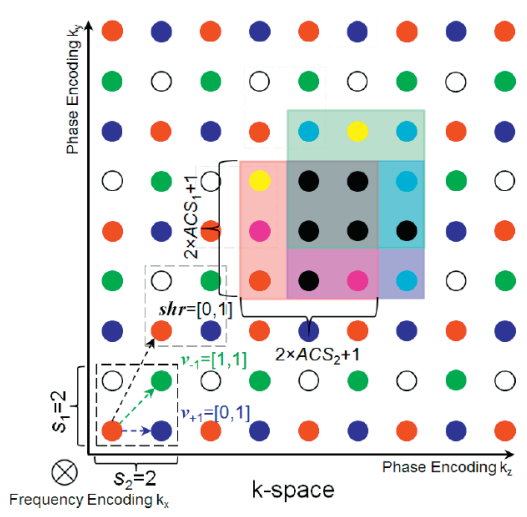

(b) An illustration of the k-space pattern with pattern parameters.

$$
\begin{aligned}
& \text { - SE signals } \\
& \text { : GRE. }{ }_{1} \text { signals } \\
& \text { - } G R E_{+1} \text { signals } \\
& \text { : SE and GRE } \text { - }_{1} \text { signals }
\end{aligned}
$$

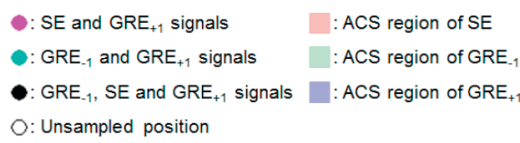

Fig. 2. An example of the proposed APIR4GRASE sampling pattern. Note the position of the ACS region is shifted along with the whole k-space. 
Table 1

Acquisition parameters. Note that the echo train length includes several skipped echoes at the beginning of each ET. The number of skipped echoes are specified in this table.

\begin{tabular}{|c|c|c|c|}
\hline & Full acquisition of phantom for retrospective experiment & Prospective optimal patterns acquisition of phantom & In-vivo acquisition \\
\hline RF pulses & Non-slice selective & Non-slice selective & Non-slice selective \\
\hline Repetition time (TR) (ms) & 400 & 1000 & 2800 \\
\hline Effective echo time (TE) (ms) & 126.1 & 205.4 & 122.2 \\
\hline Effective TE for GRE (ms) & 2.796 & 1.976 & 1.104 \\
\hline Echo train length (ETL) & 26 & 56 & 56 \\
\hline Number of skipped echoes & 3 & 3 & 3 \\
\hline Echo spacing (ESP) (ms) & 9.7 & 7.9 & 4.7 \\
\hline EPI factor (E) & 3 & 3 & 3 \\
\hline Acquisition matrix & $256 \times 256 \times 256$ & $256 \times 256 \times 256$ & $128 \times 128 \times 128$ \\
\hline FOV $\left(\mathrm{mm}^{3}\right)$ & $205 \times 205 \times 205$ & $205 \times 205 \times 205$ & $205 \times 205 \times 205$ \\
\hline Voxel size $\left(\mathrm{mm}^{3}\right)$ & $0.8 \times 0.8 \times 0.8$ & $0.8 \times 0.8 \times 0.8$ & $1.6 \times 1.6 \times 1.6$ \\
\hline Receive bandwidth $(\mathrm{kHz})$ & \pm 83.33 & \pm 83.33 & \pm 83.33 \\
\hline Scan time with full k-space pattern (min) & 19.0 & 20.6 & 14.4 \\
\hline
\end{tabular}

region is shifted along with the whole k-space.

\subsection{APIR4GRASE reconstruction}

\subsubsection{Kernel computation and signal calibration}

As each echo type has the same k-space pattern, a periodic cell with the size $s$ in two PE directions can be extracted, as shown in the dotted square in Fig. 2. For reconstruction, each relative index $\boldsymbol{p}$ in one unit cell has to be considered separately. The k-space position in PE1, PE2 and frequency encoding (FE) dimensions is represented by $\boldsymbol{k}=\left[k_{P E 1}, k_{P E 2}, k_{F E}\right] \in \mathbb{Z}^{3}$. For each $\boldsymbol{k}, \boldsymbol{p}$ can be computed as follows:

$\boldsymbol{p}(\boldsymbol{k})=\left[\begin{array}{l}k_{P E 1} \\ k_{P E 2}\end{array}\right]-\left[\begin{array}{ll}s_{1} & s h r_{1} \\ s h r_{2} & s_{2}\end{array}\right]\left\lfloor\left[\begin{array}{ll}s_{1} & s h r_{1} \\ s h r_{2} & s_{2}\end{array}\right]^{-1}\left[\begin{array}{l}k_{P E 1} \\ k_{P E 2}\end{array}\right]\right\rfloor$

Here, $\lfloor\boldsymbol{A}\rfloor$ represents the floor operation on each element of vector $\boldsymbol{A}$. For echo type $j$ and channel $c$, the signal $S_{j, c}$ at an unsampled position $\boldsymbol{k}$ can be computed as $[14,11]$

$S_{j, c}(\boldsymbol{k})=\sum_{j^{\prime}} \sum_{c^{\prime}} \sum_{\boldsymbol{b} \in \boldsymbol{B}_{j^{\prime}, \boldsymbol{p}(\boldsymbol{k})}} n_{j, c, \boldsymbol{p}(\boldsymbol{k})}\left(j^{\prime}, c^{\prime}, \boldsymbol{b}\right) \cdot S_{j^{\prime}, c^{\prime}}(\boldsymbol{k}-\boldsymbol{b})$,

where $j^{\prime}$ and $c^{\prime}$ loop over all possible $j$ and $c$, respectively; $n_{j, c, p(k)}$ encodes the convolution kernels; $\boldsymbol{B}_{j^{\prime}, \boldsymbol{p}(\boldsymbol{k})}$ is the set of offsets $\boldsymbol{b} \in \mathbb{Z}^{3}$ from $\boldsymbol{k}$ to the regularly sampled positions included in the kernel $n_{j, c, p(k)}$.

Substituting the lines in the ACS region for $S_{j, c}$ in Eq. (3) results, for each $j, c, p$, in a system of equations linear in $n\left(j^{\prime}, c^{\prime}, \boldsymbol{b}\right)$ which can be solved with linear least squares [14] possibly with Tikhonov regularization. Subsequently, all unsampled positions are reconstructed by Eq. (3), using these $n$ and all acquired data $S$ [14].

\subsubsection{Image reconstruction}

After reconstruction of the unsampled positions, the k-space of each echo type $j$ and each channel $c$ is reconstructed individually by

$I_{j, c}(x)=\operatorname{iFFT}_{k}\left(S_{j, c}(\boldsymbol{k})\right)$,

where iFFT is the inverse Fourier transform and $x \in \mathbb{Z}^{3}$ is the voxel index. Image $I_{j}$ is computed as the root mean squares (RMS) of its channel images [14]:

$I_{j}(\mathbf{x})=\sqrt{\frac{\sum_{c}\left|I_{j, c}(\mathbf{x})\right|^{2}}{N_{c}}}$,

where $N_{c}$ is the number of channels. Additionally, a combined RMS image is reconstructed by [11]

$I(\mathbf{x})=\sqrt{\frac{\sum_{j}\left|I_{j}(\mathbf{x})\right|^{2}}{E}}$.

\subsubsection{Theoretical support}

An APIR method, such as GRAPPA/ARC, obtains coil sensitivity information from a fully sampled autocalibration region in the center of $\mathrm{k}$-space. As coil relative sensitivities are smoothly changing in the image domain, they can be modelled as small convolution kernels in $\mathrm{k}$ space. Below, we argue that the differences between SE and GREs can also be encoded into the kernels and the assumptions will be investigated in the experiments.

The GREs acquired next to the SE in GRASE are attenuated by $T_{2}$ * decay and off-resonance effects. Specifically, the magnetization of echo type $j$ with the time offset $T_{j}$ (see Fig. 1) from the SE in channel $c$ can be approximated as

$S_{j, c}(\mathbf{x})=M(\mathbf{x}) C_{c}(\mathbf{x}) e^{-\left|T_{j}\right| / T_{2}{ }^{*}(\mathbf{x})-i \omega_{0}(\mathbf{x}) T_{j}}$,

where $\mathrm{M}(\mathrm{x})$ is the transverse magnetization, $C_{c}$ is the coil sensitivity of channel $c$, and $\omega_{0}$ is the off-resonance frequency.

When we regard the additional contrast images as virtual coil channels [16] with $C_{j, c}(\mathbf{x})=C_{c}(\mathbf{x}) e^{-\left|T_{j}\right| / T_{2}^{*}(\mathbf{x})-i \omega_{0}(\mathbf{x}) T_{j}}$ as the sensitivity of the echo type $j$ in channel $c$, the signal of echo type $j$ in channel $c$ can be rewritten as

$S_{j, c}(\mathbf{x})=M(\mathbf{x}) C_{j, c}(\mathbf{x})$,

therefore, just like the coil relative sensitivities, the $T_{2}$ * decay and offresonance effects can be encoded by the learned convolution kernels $n_{j, c, p(\boldsymbol{k})}$ in Eq. (3). Note that the assumed spatial smoothness of $C_{j}$ is typically preserved when $\left|T_{j}\right| \ll T_{2}{ }^{*}$ because $\omega_{0}$ is smoothly varying.

\subsection{Optimal patterns searching using phantom acquisition}

We propose the optimal k-space patterns of APIR4GRASE with different subsampling factors from retrospective exhaustive searching. Two full k-spaces $\left(E=3, \boldsymbol{s}=[1,1], \boldsymbol{s h} r=[0,0], v_{i}=[0,0] \forall i\right)$ of the ACR-NEMA MRI Phantom [18] were acquired by two acquisitions in the same session with the 3D-GRASE sequence. A 3T General Electric Discovery MR750 clinical scanner (General Electric Medical Systems, Waukesha, WI) and an eight-channel birdcage-like receive brain coil (8HRBRAIN) were used. The scan parameters are shown in the left column of Table 1 . The scan time for each full acquisition is $19.0 \mathrm{~min}$. A variable flip angle algorithm [11] was used to stabilize the amplitude of the magnetization along the ET. For each echo type, the view ordering follows a $T_{2}$ weighted linear encoding trajectory as described by $[7,8]$. The cylindrical phantom was placed axially in the coil array, with S/I the frequency encoding direction.

All possible k-space patterns with $\|\boldsymbol{s}\|_{\infty} \leqslant 4, s h r_{1}<s_{1}, s h r_{2}<s_{2}$ and $\|\boldsymbol{s h} \boldsymbol{r}\|_{0} \leqslant 1,\|\boldsymbol{v}\|_{\infty} \leqslant 2$ for both $\mathrm{GRE}_{-1}$ and $\mathrm{GRE}_{+1}, A C S_{1}=A_{C S}=12$ were retrospectively constructed from the first full acquisition, and 
subsequently reconstructed by APIR4GRASE; hence, evaluating 40,000 patterns in total. Note that not all patterns are unique, but we did not remove these duplications in the evaluation. For the pattern search the size of the convolution kernel of APIR4GRASE was [5,5,1] in PE1, PE2 and FE dimensions in k-space for every $\boldsymbol{p}$.

The mean square error (MSE) of each reconstructed image was evaluated. As reference, we used the image with the corresponding contrast reconstructed from the second full acquisition. For SORT GRASE, the reference is the an image reconstructed from the fully sampled SE k-space of the second full acquisition. A small region in the phantom with non-stationary bubbles was excluded in the MSE computation by a manual mask. The MSE of the first full acquisition was also computed with the reference of the second full acquisition to estimate the noise contribution. Under each distinct subsampling factor of $\boldsymbol{s}$, the k-space pattern with the lowest mean of MSE of all echo types was selected as the optimal pattern.

\subsection{Prospective evaluation of optimal patterns with phantom acquisition}

To prospectively verify the reconstruction quality of the selected optimal k-space patterns, each of them was used in a second scan session, with the same scanner and coil, using the same phantom as in the first experiment. The scan parameters in this session are shown in the middle column of Table 1 . As reference, two full k-spaces were acquired as well. The scan time for the full acquisition is $20.6 \mathrm{~min}$. For each optimal pattern, the total subsampling factor and the scan time are shown in Table 2.

Besides the APIR4GRASE reconstruction, the GRAPPA reconstruction was also performed on the k-space of each individual echo type for comparison. The convolution kernel has a size of $[5,5,5]$ for both APIR4GRASE and GRAPPA. Tikhonov regularization [19] was added to the kernel estimation to avoid excessive noise amplification with the increased kernel size. The regularization parameter was firstly narrowed from a wide range based on the MSE, and a single value, identical for all patterns, was selected based on visual inspection of the reconstructed images, trying to avoid significant presence of both artifacts and noise.

For comparison, the conventional SORT GRASE k-space was retrospectively constructed from the first full acquisition. This avoids eddy current induced artifacts due to larger phase encode steps between SE and GRE of a prospective SORT acquisition.

To compare the performance of APIR4GRASE, GRAPPA and conventional SORT GRASE, the MSE of their reconstructed images was computed. To also compare the noise suppression ability of APIR4GRASE and GRAPPA, the g-factor map was computed with the pseudo multiple replica method [20] with 300 iterations by adding Gaussian white noise in the acquired k-space. The magnitude level of

Table 2

$\boldsymbol{s h} \boldsymbol{r}, \boldsymbol{v}_{-1}, \boldsymbol{v}_{+1}$, total subsampling factors, and scan times of the prospective phantom acquisitions for the optimal patterns.

\begin{tabular}{llllll}
$\boldsymbol{s}$ & $\boldsymbol{s h} \boldsymbol{r}$ & $\boldsymbol{v}_{-1}$ & $\boldsymbol{v}_{+1}$ & Total subsampling factor & Scan time (min) \\
\hline$[1,2]$ & {$[0,1]$} & {$[-2,2]$} & {$[-2,1]$} & 2 & 10.4 \\
{$[1,3]$} & {$[0,1]$} & {$[2,2]$} & {$[-2,2]$} & 3 & 7.0 \\
{$[1,4]$} & {$[0,2]$} & {$[2,2]$} & {$[2,1]$} & 4 & 5.3 \\
{$[2,1]$} & {$[1,0]$} & {$[-2,2]$} & {$[-2,1]$} & 2 & 10.4 \\
{$[2,2]$} & {$[0,1]$} & {$[1,-1]$} & {$[1,-2]$} & 4 & 5.3 \\
{$[2,3]$} & {$[1,0]$} & {$[1,1]$} & {$[2,2]$} & 6 & 3.6 \\
{$[2,4]$} & {$[0,1]$} & {$[0,2]$} & {$[1,1]$} & 8 & 2.7 \\
{$[3,1]$} & {$[1,0]$} & {$[2,2]$} & {$[-2,2]$} & 3 & 7.0 \\
{$[3,2]$} & {$[1,0]$} & {$[1,2]$} & {$[0,2]$} & 6 & 3.6 \\
{$[3,3]$} & {$[1,0]$} & {$[2,2]$} & {$[2,0]$} & 9 & 2.5 \\
{$[3,4]$} & {$[0,0]$} & {$[1,-2]$} & {$[2,-1]$} & 12 & 1.9 \\
{$[4,1]$} & {$[2,0]$} & {$[2,1]$} & {$[2,2]$} & 4 & 5.3 \\
{$[4,2]$} & {$[1,0]$} & {$[-1,2]$} & {$[2,2]$} & 8 & 2.7 \\
{$[4,3]$} & {$[0,2]$} & {$[2,0]$} & {$[1,1]$} & 12 & 1.9 \\
\hline
\end{tabular}

the simulated noise was estimated by the standard deviation in the background area of the magnitude image of the first full acquisition. Both APIR4GRASE and GRAPPA include the RMS reconstruction (Eq. (5)) on the channel images, therefore, the g-factor map is also computed on the RMS image of each echo type.

\subsection{Retrospective in-vivo experiment}

In-vivo performance of the optimal patterns was evaluated by retrospectively subsampling fully acquired $\mathrm{k}$-spaces of all echo types $\left(\mathrm{GRE}_{-1}, \mathrm{SE}\right.$, and $\left.\mathrm{GRE}_{+1}\right)$. With Institutional Review Board approval and after obtaining informed consent the brain of five volunteers was scanned by the same sequence, with the same coil and scanner as in the phantom experiment. The scan parameters are shown in the right column of Table 1. The scan time for each full acquisition is $14.4 \mathrm{~min}$. The frequency encoding direction was S/I.

The k-spaces of the optimal patterns and the SORT pattern were retrospectively constructed from the full acquisition. For reference, the full k-space of each echo type was reconstructed into an image by iFFT.

To compare the performance of APIR4GRASE, GRAPPA and the conventional SORT GRASE on the in-vivo data, the MSE of their reconstructed images was computed. For the MSE computation, the full kspaces of all volunteers are scaled to the same energy level as the first volunteer. The size of the convolution kernel of both APIR4GRASE and GRAPPA is [7,7,5]. Regularization was also used in the convolution kernel computation with a single parameter for all patterns. The parameter selection method was the same as in the phantom experiment.

To validate the assumed spatial smoothness across echo types in brain imaging, we performed a phase preserved reconstruction for each echo type from $I_{j, c}$ of the full acquisition using the Walsh combining method [21], and performed voxel-wise division of the complex-valued images between all echo type pairs.

\subsection{Prospective in-vivo experiment}

Using the same acquisition setting as the retrospective in-vivo acquisition, the optimal patterns of $s=[2,3],[2,4]$, and [3,3] were acquired prospectively in one volunteer. To validate the image quality with respect to the images of the retrospective acquisitions, APIR4GRASE with the same convolution kernel size was performed to reconstruct the images of the prospective acquisition.

To validate APIR4GRASE with high subsampling factor in high resolution in-vivo imaging, a prospective T2-weighted acquisition with the optimal pattern of $s=[3,3]$ was performed in one volunteer with the settings the same with the prospective optimal patterns acquisition of phantom (the middle column) in Table 1 except that TR $=2800 \mathrm{~ms}$, $\mathrm{TE}=272.8 \mathrm{~ms}$, $\mathrm{ESP}=9.7 \mathrm{~ms}$. The resolution was $0.8 \mathrm{~mm}$ isotropic. This acquisition acquired an elliptical k-space by skipping the corners in the PE plane and had scan time of $5.5 \mathrm{~min}$. Image reconstruction was performed identically to the retrospective acquisitions. For reference, a GRASE SORT image with the same acquisition settings was also acquired. This acquisition filled the rectangular k-space with acceleration factor two in PE1 direction without skipping corners and took around $9.4 \mathrm{~min}$. The image of this acquisition was reconstructed by ARC [15].

\section{Results}

\subsection{Optimal patterns searching using phantom acquisition}

Fig. 3 (a) presents the mean of the MSEs of the reconstructed $\mathrm{GRE}_{-1}, \mathrm{SE}$ and $\mathrm{GRE}_{+1}$ images for all 40,000 tested patterns. Each block in Fig. 3 (a) contains the MSEs of all 625 tested patterns under one specific pair of subsampling factors in both $\mathrm{PE}$ directions and one specific shearing condition. Fig. 3 (b) shows how the MSEs of all patterns were organized in one block by zooming in on the block with 


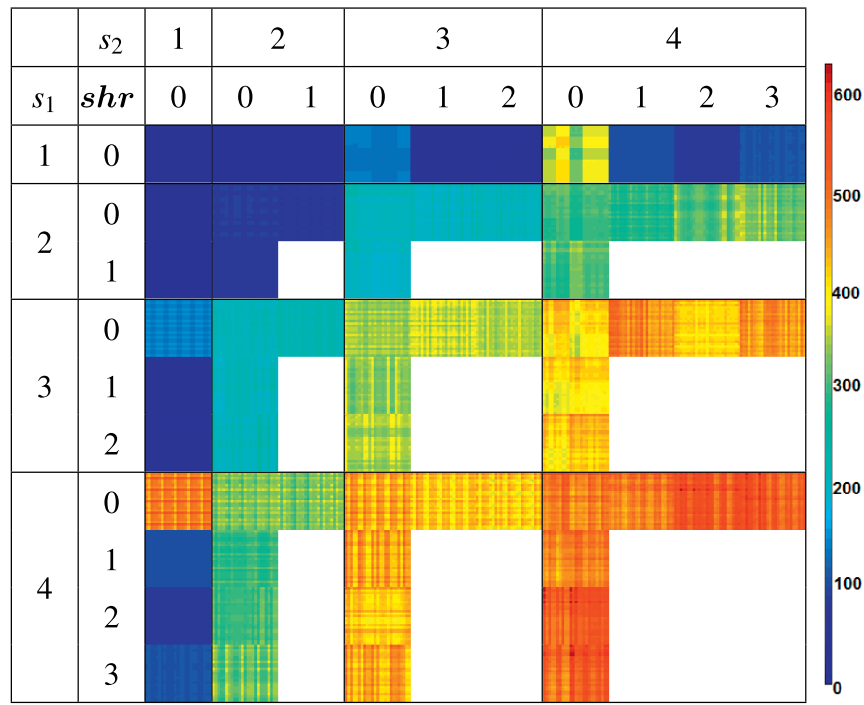

(a) The mean of MSE of $\mathrm{GRE}_{-1}, \mathrm{SE}$ and $\mathrm{GRE}_{+1}$ images of all tested patterns

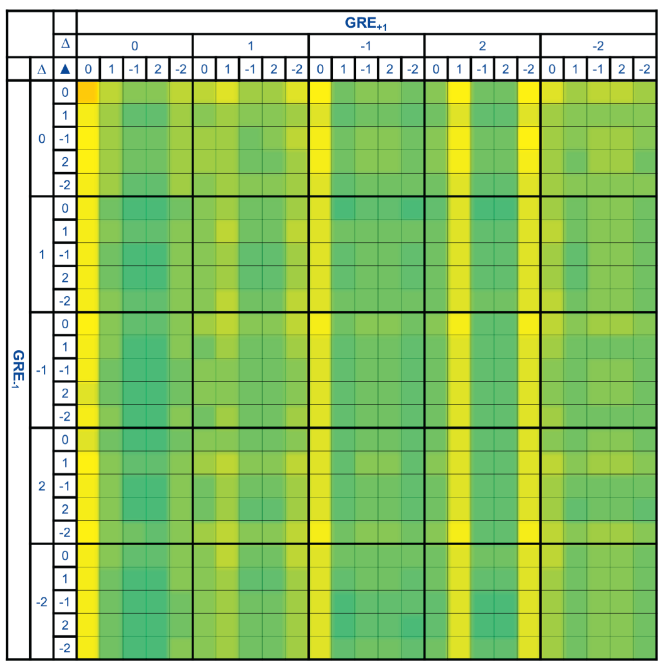

(b) Results in the block of $s=[3,3], \boldsymbol{s h r}=[1,0]$

Fig. 3. The mean of MSE of $\mathrm{GRE}_{-1}$, SE and $\mathrm{GRE}_{+1}$ images of all tested patterns (a) and a zoomed-in version showing how the results are organized in each block (b). $\Delta=\boldsymbol{v}_{-1}$ and $\boldsymbol{\Delta}=\boldsymbol{v}_{+1}$.

$\boldsymbol{s}=[4,1]$ and $\boldsymbol{s h} \boldsymbol{r}=[0,0]$. The MSEs of each individual echo type are presented in Supplementary materials ( Figs. S1-S3).

Fig. 3 (a) shows that patterns with different $s \boldsymbol{r}$ or $\boldsymbol{v}$ differ in MSE. The patterns with non-zero shearing mostly have lower MSE than their counterparts with zero shearing when only one PE direction is highly subsampled. The impact of $\boldsymbol{v}$ is higher in patterns with higher $s_{1} \times s_{2}$.

The optimal patterns selected based on the mean of MSEs of GRE ${ }_{-1}$, $\mathrm{SE}$ and $\mathrm{GRE}_{+1}$ images are shown in Fig. $4(\mathrm{a}-\mathrm{O})$. The conventional SORT GRASE pattern is also shown in Fig. 4 (p). Note that the optimal pattern with $s=[1,2]$ is identical to the optimal pattern with $s=[2,1]$, and the optimal pattern with $s=[1,3]$ is identical to the optimal pattern with $\boldsymbol{s}=[3,1]$. The detailed parameters of $\boldsymbol{s h}, \boldsymbol{v}_{-1}, \boldsymbol{v}_{+1}$, and the total subsampling factors for the optimal patterns are shown in Table 2.

Fig. 5 (a) shows the mean of the MSE of the three echo types with the optimal pattern under each subsampling factor $s_{1} \times s_{2}$. Different points under an identical total subsampling factor represent different patterns (e.g. $\boldsymbol{s}=[2,3]$ vs $\boldsymbol{s}=[3,2]$ ). The black line in Fig. 5 (a) shows the theoretically expected lower bound on the MSE for each subsampling factor, calculated by multiplying the MSE of the repeated full acquisition, $\mathrm{MSE}_{\text {rep }}$, by the subsampling factor, i.e., $\mathrm{MSE}_{\text {rep }} \times s_{1} \times s_{2}$. However, as is well known for parallel imaging, MSE increases further due to aliasing artifacts and noise amplification (g-factor) in practice. Above the subsampling factor of four, the MSE of the optimal patterns exceeds the black line, indicating that noise amplification or the introduction of parallel imaging artifacts substantially contribute to the MSE.

\subsection{Prospective evaluation of optimal patterns with phantom acquisition}

Fig. 6 (a-c) shows, for several optimal patterns $\left(s_{1} \in\{1,2,3,4\}\right.$ and $s_{2}=3$ ), one slice in the two PE dimensions of the reconstructed combined images, the SE images of the prospective phantom acquisition by APIR4GRASE, and the SE images by GRAPPA. The corresponding $g$ factor maps of the SE image reconstructions are shown in Fig. 6 (d, e). The results of all echo types with all optimal patterns are presented in Supplementary materials ( Figs. S4-S15). Fig. 6 (a-c) shows more artifacts for the higher subsampled patterns. Compared to the GRAPPA SE image, the APIR4GRASE combined image and the SE image show less artifacts under each subsampling factor. In Fig. 6 (d, e), the g-factor of APIR4GRASE reconstruction is also lower than the GRAPPA reconstruction.

Fig. 5 (b) presents the mean of the MSE of the three echo types of both APIR4GRASE and GRAPPA reconstructed images for each of the optimal patterns. The black line in Fig. 5 (b) has the same meaning as in Fig. 5 (a). As can be seen, the MSE of APIR4GRASE is lower than GRAPPA, which is more obvious for subsampling factors $s_{1} \times s_{2} \geqslant 6$. The overall better MSE of APIR4GRASE in Fig. 5 (b) than in Fig. 5 (a) is probably due to the contribution of the Tikhonov regularization.

Fig. 7 (a) shows the SORT GRASE image and Fig. 7 (b) the APIR4GRASE combined image with the optimal k-space pattern of $s_{1}=1$ and $s_{2}=3$, and their MSEs. The scan time of APIR4GRASE is $1 \%$ longer than SORT GRASE due to the fully acquired ACS region. The modulation artifacts present in SORT GRASE image are not present in APIR4GRASE images. Additionally, from the same data, APIR4GRASE reconstructs the SE as well as the GRE images shown in Fig. 7 ( c-e). The MSE of the APIR4GRASE combined image is 78\% lower than the MSE of the SORT GRASE image. The oscillatory pattern in the lower part of the APIR4GRASE images, as shown inside the blue ellipse in Fig. 7 (b), is also present in the fully sampled SE image. Hence this is not an artifact introduced by the inclusion of gradient echoes nor the subsampling but intrinsic in the VFA-FSE sequence and trajectory used. It is probably caused by a strong structure pattern at few slice distance in the phantom.

\subsection{Retrospective in-vivo experiment}

Fig. 8 shows one axial slice of the reconstructed APIR4GRASE combined brain images (a), the APIR4GRASE SE images (b), and the GRAPPA SE images (c), with several scanned optimal patterns $\left(s_{1}\right.$ $\in\{1,2,3,4\}$ and $s_{2}=3$ ) for the first retrospective volunteer scan. The left half of these images contains the reconstructed image and the right half the difference with the fully sampled reference image. The g-factor maps of APIR4GRASE and GRAPPA on the SE image reconstruction are shown in Fig. 8 (d, e). These maps clearly show that APIR4GRASE has a lower noise amplification than GRAPPA when $s_{1} \times s_{2} \geqslant 6$. For this volunteer the results of all echo types with all optimal patterns are presented in Supplementary materials ( Figs. S16-S27) and the results of the other volunteers are presented in the Supplementary material (Figs. S28-S31).

Fig. 5 (c) presents the MSE results of both APIR4GRASE and GRAPPA reconstructions on the in-vivo data for the five volunteer scans. For each volunteer, the MSE of GRAPPA is increasingly higher than APIR4GRASE when the subsampling factor increases, and the difference is most clear when $s_{1} \times s_{2} \geqslant 8$.

Fig. 9 shows the in-vivo images of the SORT GRASE (a) and 


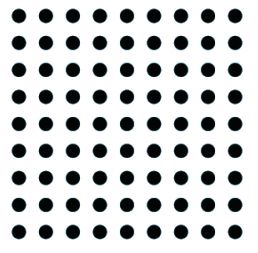

(a) $\boldsymbol{s}=[1,1]$

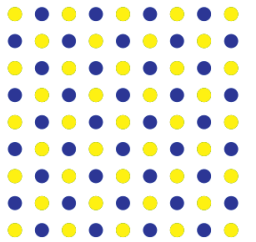

(e) $s=[2,1]$

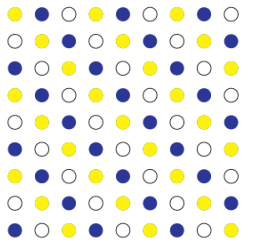

(i) $s=[3,1]$

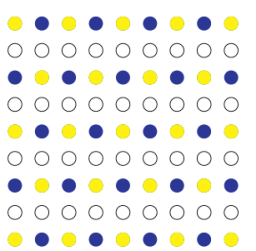

(m) $s=[4,1]$

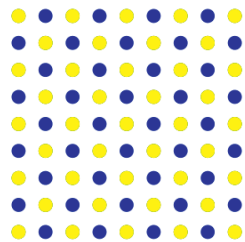

(b) $s=[1,2]$

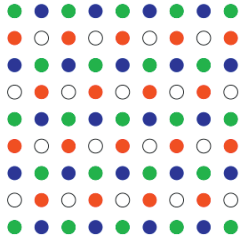

(f) $\boldsymbol{s}=[2,2]$

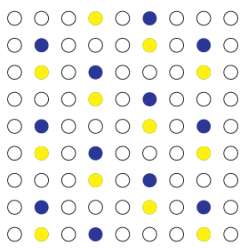

(j) $s=[3,2]$

$0 \cdot 000 \cdot 0 \cdot 0$ $0 \bullet 0 \bullet 000 \bullet 0$ $0 \circ 0 \bullet 0 \bullet 000$ $000 \bullet 0 \cdot 0 \cdot 0$ $0 \bullet 000$ $0 \bullet 0 \bullet 000 \cdot 0$ 0000000 000 $0,000 \cdot 0 \cdot 0$

(n) $s=[4,2]$

: SE signals

- $\mathrm{GRE}_{-1}$ signals

: $\mathrm{GRE}_{+1}$ signals

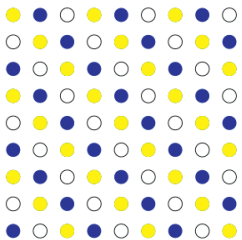

(c) $s=[1,3]$

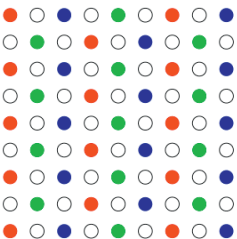

(g) $s=[2,3]$

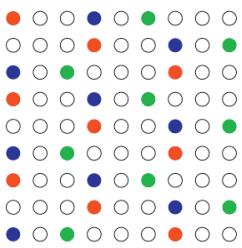

(k) $s=[3,3]$

$0 \cdot 00 \cdot 00 \cdot 0$ - $00 \cdot 00 \cdot 00$ 000000000 $00 \bullet 00 \bullet 00$ - $00 \bullet 00 \bullet 00$ $0 \circ \bullet \circ, \bullet \circ ०$ 000000000 $\circ \bullet \circ, \bullet \circ \bigcirc \bullet 0$

$00 \bullet 00 \bullet 00$

(o) $s=[4,3]$

: SE and GRE signals

: $\mathrm{GRE}_{-1}, \mathrm{SE}$ and $\mathrm{GRE}_{+1}$ signals

$O$ : Unsampled position

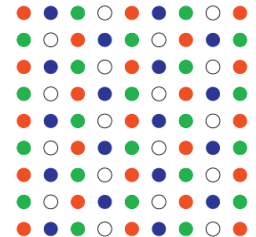

(d) $\boldsymbol{s}=[1,4]$

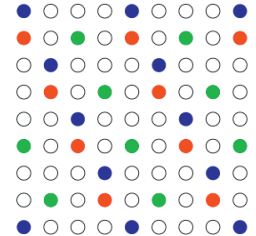

(h) $s=[2,4]$

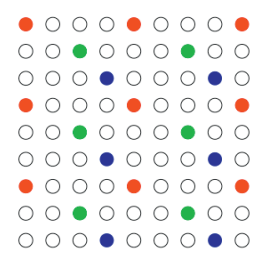

(1) $s=[3,4]$

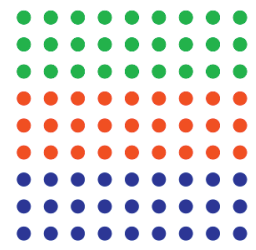

(p) SORT GRASE

Fig. 4. Optimal patterns for APIR4GRASE (a-o) and the conventional SORT GRASE pattern (p).

APIR4GRASE images (b-e) for the first volunteer and their corresponding MSEs in the brain region. The MSE calculation excluded the skull and background regions, since they are not essential for diagnosis. In the brain region, segmented using FSL (version 5.0.2.2) [22], the MSE was reduced by $94 \%$. Despite lying outside the brain, it is worth pointing out the modulation artifacts around the skull in the SORT GRASE image, which were largely eliminated by APIR4GRASE.

Fig. 10 shows the real (first row) and imaginary (second row) part of the logarithm of the voxel-wise division between the complex-valued images of the different echo types. The real component represents the magnitude ratio and the imaginary part represents the phase difference. The sagittal plane, instead of the axial plane, is shown as it contains more variation. Observe that these ratio images are spatially smooth in the brain region.

\subsection{Prospective in-vivo experiment}

Fig. 11 shows the combined images of the retrospective acquisition (first row) and the prospective acquisition (second row) with the optimal patterns of $s=[2,3],[2,4]$, and [3,3] from left to right. The images show similar subsampling artifacts.

Fig. 12 shows the images of the high resolution prospective acquisitions for both APIR4GRASE (a) and the SORT GRASE (b). With reduced scan time, APIR4GRASE achieved image quality similar to the SORT GRASE.

\section{Discussion}

We proposed APIR4GRASE to reduce modulation artifacts in conventional GRASE using the correlation among SE and GRE through autocalibrated parallel imaging reconstruction [16,14]. The application of the subsampling pattern search method showed substantial differences in MSE among patterns with identical subsampling factor. The optimal patterns that were prospectively validated show that with similar acquisition time, APIR4GRASE is able to avoid modulation artifacts in conventional SORT GRASE. Additionally, APIR4GRASE can simultaneously reconstruct GRE images with similar image quality. This might be useful for detection of hemorrhage, microcalcifications or iron deposits [23]. $T_{2}^{*}$ weighting can be enhanced by increasing $E$, which increases the maximum $\left|T_{j}\right|$. By combining the SE and GRE images using RMS, a combined image with better quality and lower MSE is achieved.

A current limitation of APIR4GRASE is that phase images are not reconstructed due to RMS reconstruction. Another limitation is the assumption of small or spatially smooth $T_{2}^{*}$ decay during $T_{j}$, though this assumption is typically satisfied in brain imaging. The potential of 


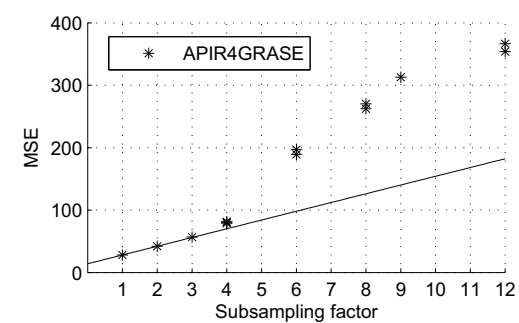

(a) The lowest mean of the MSE of $\mathrm{GRE}_{-1}, \mathrm{SE}$ and $\mathrm{GRE}_{+1}$ images of the phantom with retrospectively subsampled patterns under each total subsampling factor.

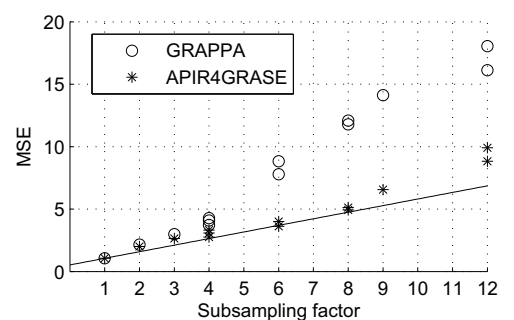

(b) The mean of the MSE of $\mathrm{GRE}_{-1}$, SE and $\mathrm{GRE}_{+1}$ images of the phantom with prospective acquired optimal patterns.

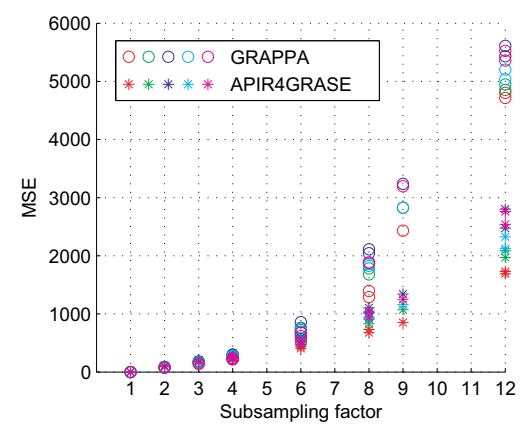

(c) The mean of the MSE of $\mathrm{GRE}_{-1}, \mathrm{SE}$ and $\mathrm{GRE}_{+1}$ images with retrospectively subsampled optimal patterns on the in-vivo data. Different colors indicate different volunteers.

Fig. 5. The MSE of the reconstructed images. Note that the subsampling factor is defined by $s_{1} \times s_{2}$, where the effect of the ACS region is neglected.
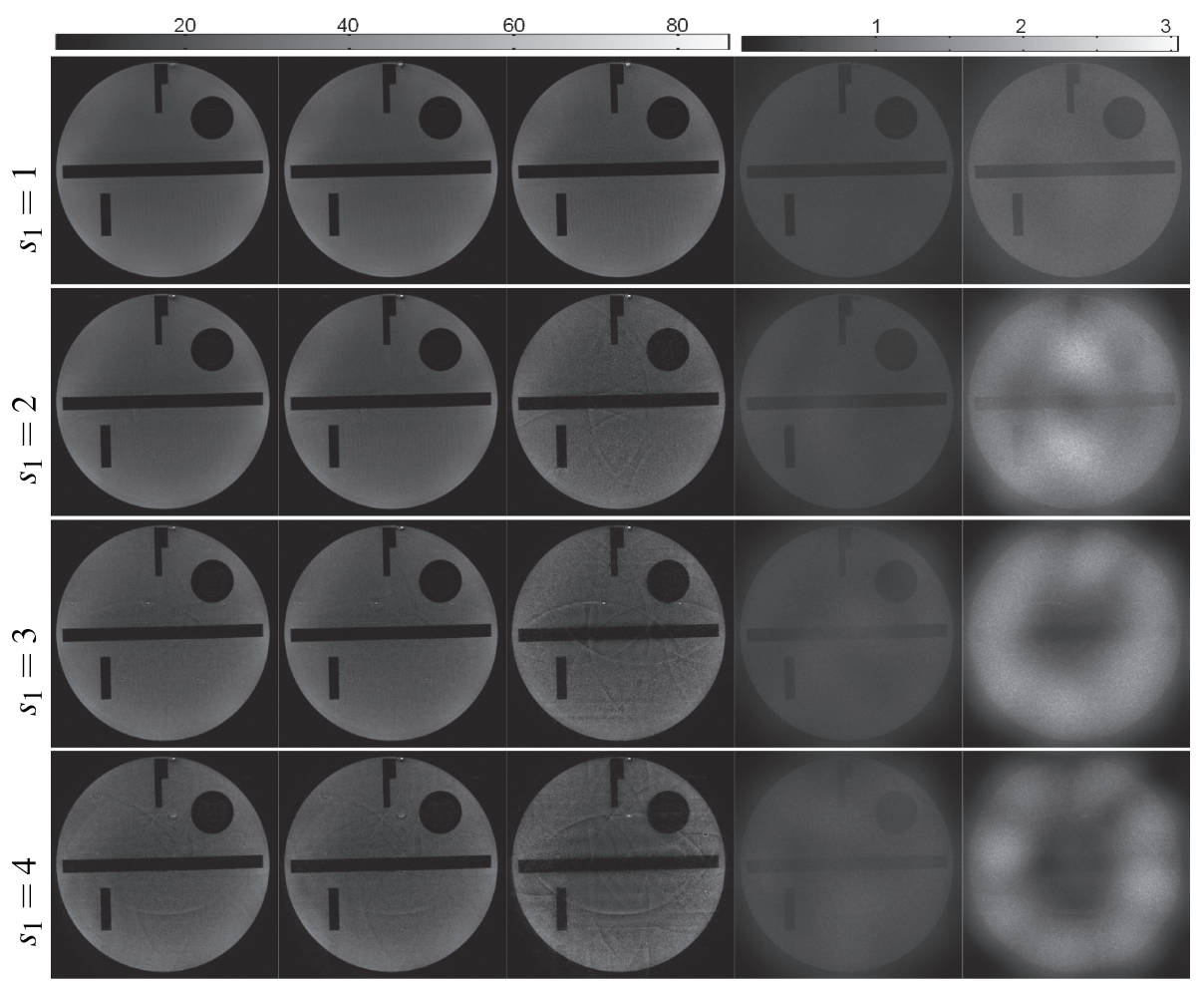

(a) (c)
Fig. 6. The images and the g-factor maps of some prospectively acquired optimal patterns $\left(s_{1}\right.$ $\in\{1,2,3,4\}$ and $s_{2}=3$ ) of the phantom acquisition with APIR4GRASE and GRAPPA reconstruction. For each of them, one axial slice (the PE1 $\times$ PE2 plane) is shown. (a) The combined image of APIR4GRASE; (b) The SE image of APIR4GRASE; (c) The SE image of GRAPPA; (d) The g-factor map of APIR4GRASE on SE; (e) The $g$-factor map of GRAPPA on SE. 


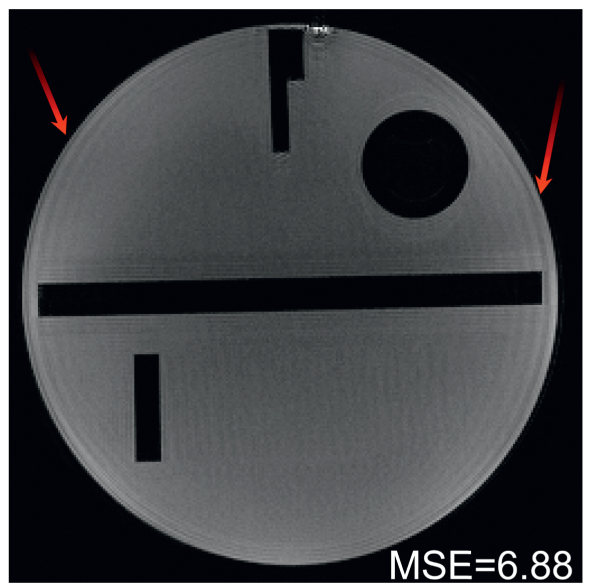

(a) The SORT GRASE image

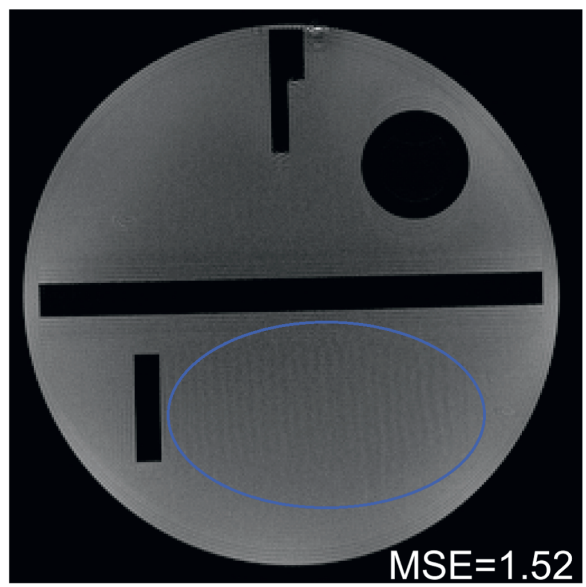

(b) APIR4GRASE combined image
Fig. 7. The comparison of the SORT GRASE image (a) and the APIR4GRASE images (b-e) of the prospective phantom acquisition with the optimal kspace pattern with $s=[1,3]$. The red arrows point to the modulation artifacts in the SORT GRASE image. The blue ellipse indicates some ringing artifacts which are not introduced by the inclusion of gradient echoes nor the subsampling but intrinsic in the VFA-FSE sequence and trajectory used. (c-e) show the APIR4GRASE image of each echo type. (For interpretation of the references to color in this figure legend, the reader is referred to the web version of this article.)

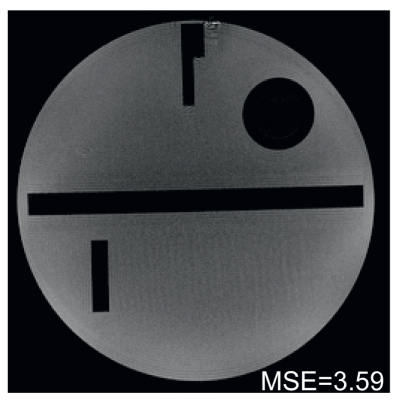

(c) $\mathrm{GRE}_{-1}$ image

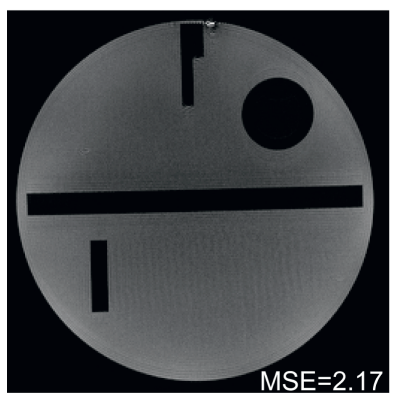

(d) SE image

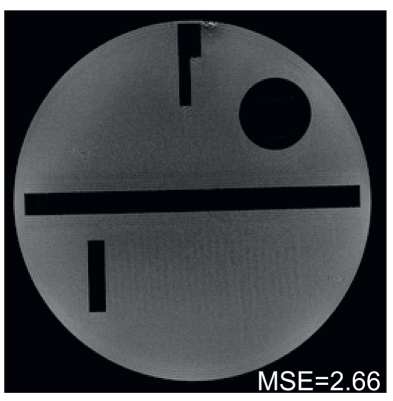

(e) $\mathrm{GRE}_{+1}$ image
APIR4GRASE in anatomies with a relative short $T_{2}^{*}$ decay, e.g. knee, requires further investigation.

For the subsampled patterns, besides the specific subsampling factor, the k-space pattern also affects the performance of APIR4GRASE. This effect is stronger for higher subsampling factors. To achieve optimal reconstruction quality, the optimal patterns with $s_{1} \times s_{2} \leqslant 12$ were selected by a full search over all possible patterns. By testing sheared and shifted sampling grids of the different echo types, our exhaustive search identified optimal sampling patterns that covered the unit cell more or less uniformly with low discrepancy. The optimal

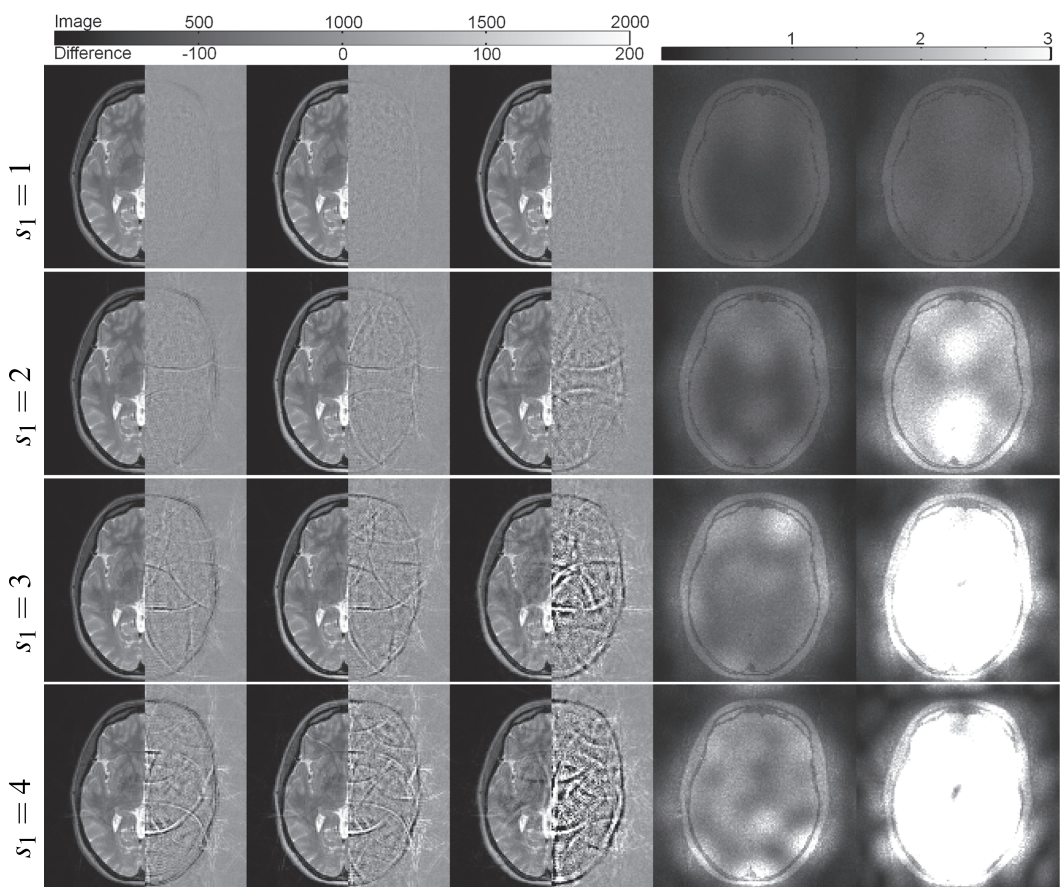

(a) (b) (c) (d)
Fig. 8. The images and the g-factor maps of some retrospectively constructed optimal patterns $\left(s_{1} \in\{1,2,3,4\}\right.$ and $\left.s_{2}=3\right)$ of the first in-vivo acquisition with APIR4GRASE and GRAPPA reconstruction. For each of them, one axial slice (the PE1 $\times$ PE2 plane) is shown. (a) The combined image of APIR4GRASE; (b) The SE image of APIR4GRASE; (c) The SE image of GRAPPA; (d) The g-factor map of APIR4GRASE on SE; (e) The g-factor map of GRAPPA on SE. In (a-c), the left half contains the reconstructed image and the right half the difference with the fully sampled reference image. 


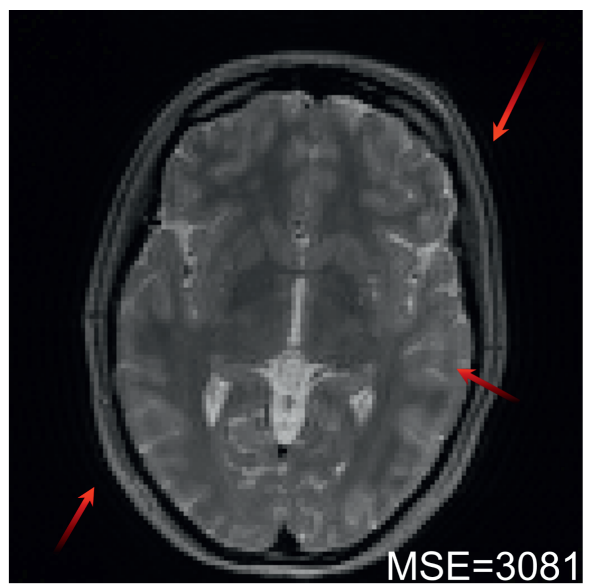

(a) The SORT GRASE image

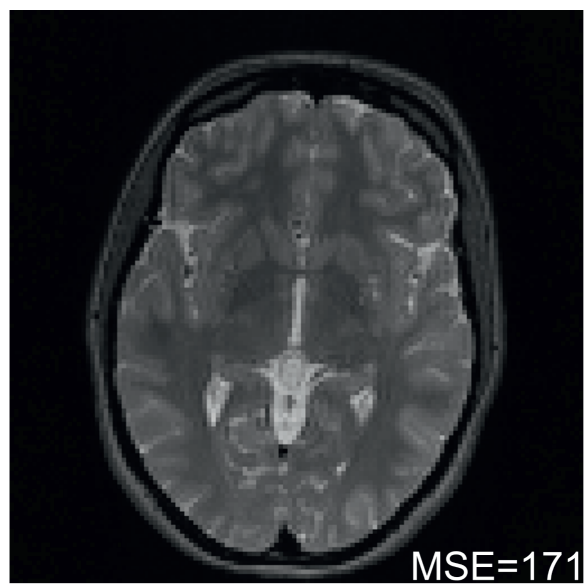

(b) APIR4GRASE combined image

Fig. 9. The comparison of the SORT GRASE image (a) and the APIR4GRASE images (b-e) of the first retrospective in-vivo acquisition with the optimal k-space pattern with $s=[1,3]$. The red arrows point to the modulation artifacts in the SORT GRASE image. Note the clearly visible ring of the skull is the first lobe of a series of rings. (c-e) show the APIR4GRASE image of each echo type. (For interpretation of the references to color in this figure legend, the reader is referred to the web version of this article.)

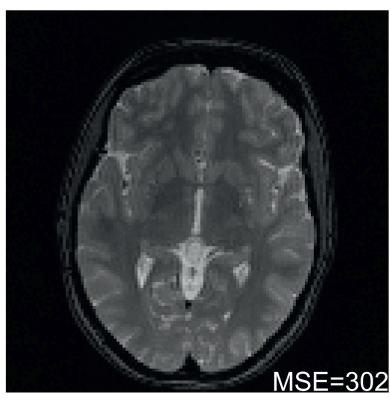

(c) GRE-1 image

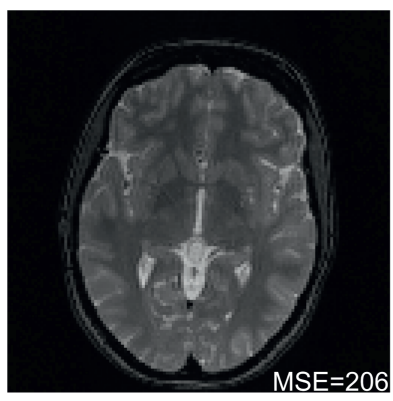

(d) SE image

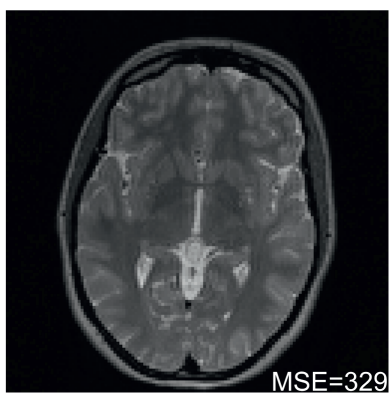

(e) $\mathrm{GRE}_{+1}$ image

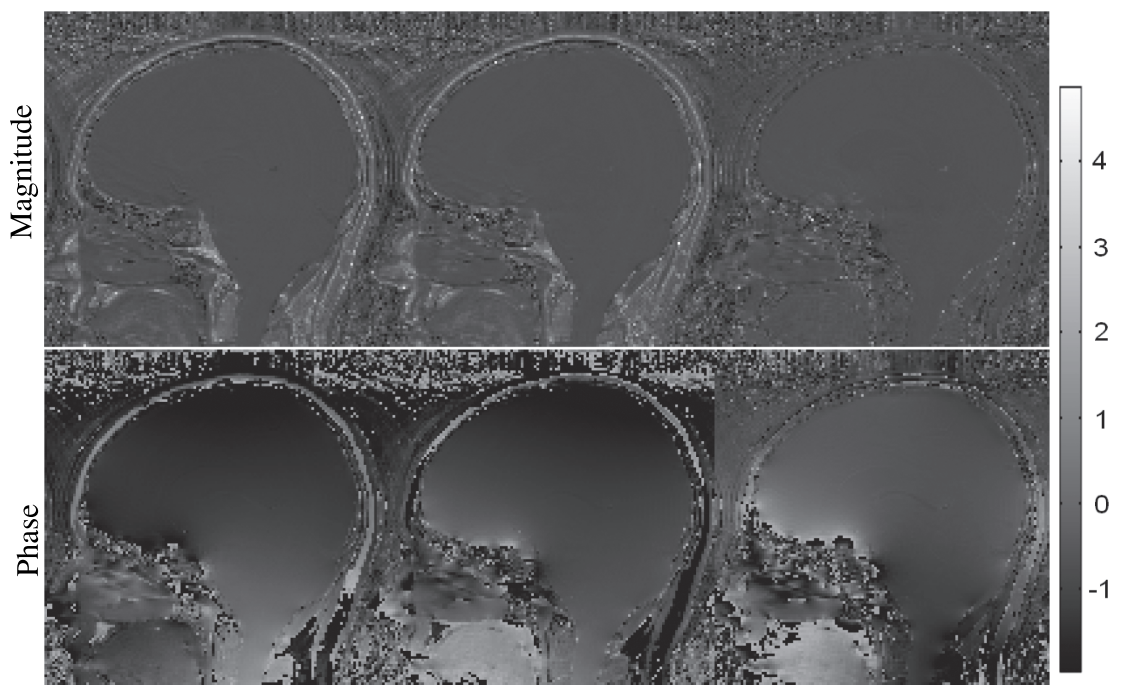

(a) (b)
Fig. 10. The voxel-wise division of the phase reserved reconstruction between two different echo types in the low resolution in-vivo full acquisition. (a) The spin echo image over the first gradient echo image, (b) the spin echo image over the second gradient echo image, and (c) the first gradient echo image over the second gradient echo image. patterns were validated on both phantom and in-vivo brain imaging with settings in a very different range (TR, ETL, resolution, etc.). This provides useful suggestions on the choice of k-space pattern for the APIR4GRASE acquisition with $E=3$ with a widely used eight-channel head receive coil. Since the signal correlation that APIR4GRASE explored mainly comes from coil sensitivity and smoothness between echo types, the selected patterns are expected to be nearly optimal for all the listed protocols (e.g. TR, TE, ETL, Matrix size, etc.) with the identical coil and echo types. For different $E(>3)$, or different receive coils, the pattern optimization might have to be repeated.

\section{Conclusions}

By integrating autocalibrated parallel imaging reconstruction and using selected optimized sampling patterns, APIR4GRASE enables achieving better image quality with less aliasing artifacts and noise amplification than conventional 3D-GRASE. Compared to GRAPPA which reconstructs one image, APIR4GRASE includes all echo types as virtual coil channels and reconstructs images for each echo type separately. APIR4GRASE achieves $0.8 \mathrm{~mm}$ 3D isotropic $T_{2}$-weighted brain imaging with an acceleration factor of nine, i.e., $\boldsymbol{s}=[3,3]$, resulting in an acquisition time of $5.5 \mathrm{~min}$. 


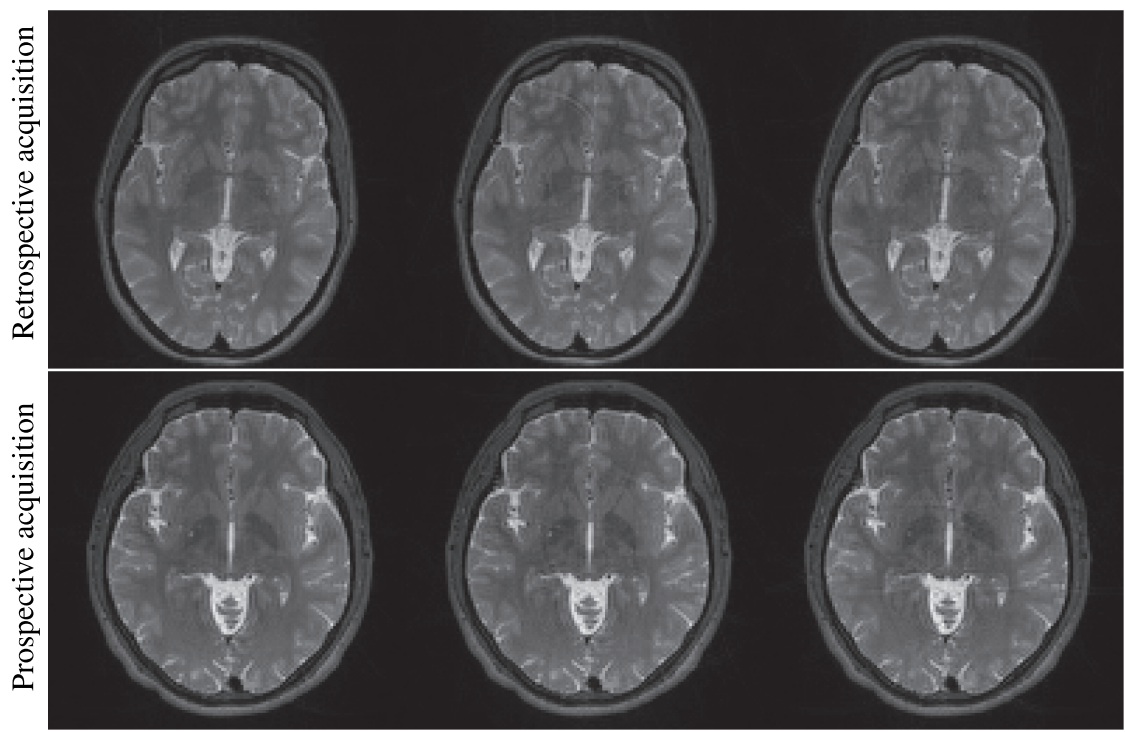

(a)

(b)

(c)

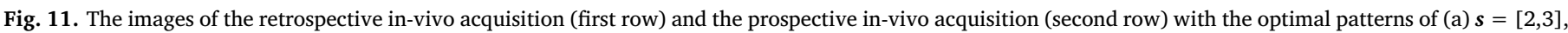
(b) $s=[2,4]$, and (c) $s=[3,3]$. Note that the prospective and retrospective datasets are acquired from different volunteers.

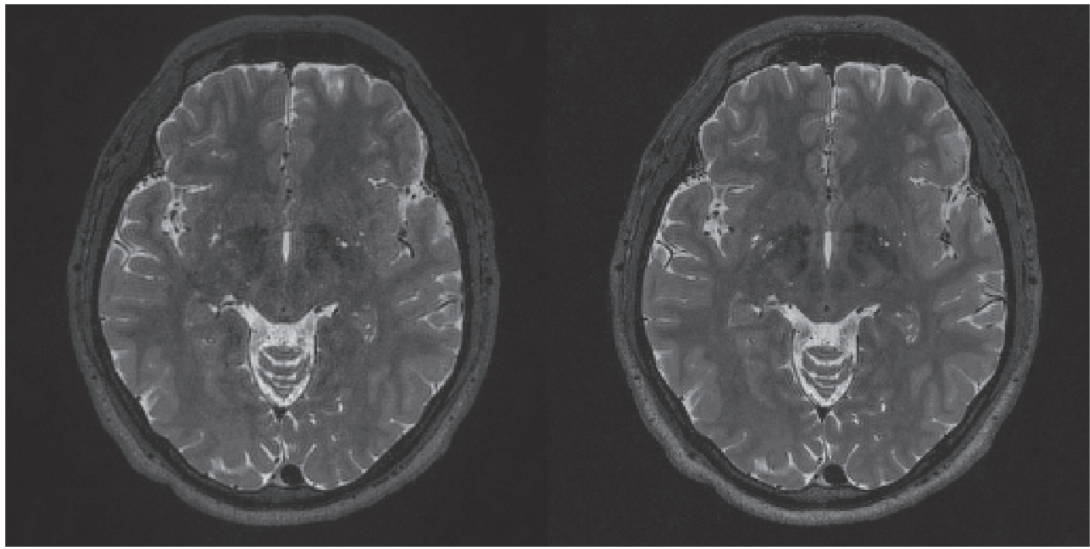

(a) The APIR4GRASE combined image

(b) The SORT GRASE image

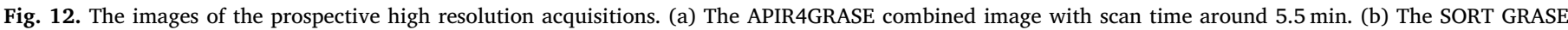
image with scan time around $9.4 \mathrm{~min}$.

\section{Acknowledgment}

This work was partially financially supported by the China Scholarship Council, Dutch Technology Foundation STW (CARISMA 11631), EUR fellowship, and General Electric Healthcare research grant (Work Statement B-GEHC-2).

\section{Appendix A. Supplementary data}

Supplementary data to this article can be found online at https:// doi.org/10.1016/j.mri.2019.08.019.

\section{References}

[1] Hennig J, Nauerth A, Friedburg H. RARE imaging: a fast imaging method for clinical MR. Magn Reson Med 1986;3(6):823-33.

[2] Michael Stehling K, Turner Robert, Mansfield Peter. Echo-planar imaging: magnetic resonance imaging in a fraction of a second. Science 1991;254(5028):43-50.

[3] Oshio Koichi, Feinberg David A. GRASE (Gradient-and spin-echo) imaging: a novel fast MRI technique. Magn Reson Med 1991;20(2):344-9.

[4] Oshio Koichi. vGRASE: separating phase and T2 modulations in 2D. Magn Reson Med 2000;44(3):383-6.
[5] Tan Huan, Scott Hoge W, Hamilton Craig A, Günther Matthias, Kraft Robert A. 3D GRASE PROPELLER: improved image acquisition technique for arterial spin labeling perfusion imaging. Magn Reson Med 2011;66(1):168-73.

[6] Ramanna S, Feinberg DA. Single-shot 3D GRASE with cylindrical k-space trajectories. Magn Reson Med 2008;60(4):976-80.

[7] Mugler John P. Improved three-dimensional GRASE imaging with the SORT phaseencoding strategy. J Magn Reson Imaging 1999;9(4):604-12.

[8] Cristobal-Huerta Alexandra, Poot Dirk HJ, Vogel Mika W, Krestin Gabriel P, Hernandez-Tamames Juan A. K-space trajectories in 3D-GRASE sequence for high resolution structural imaging. Magn Reson Imaging 2018;48:10-9.

[9] Burl Michael, Young Ian R. Eddy currents and their control. New York: Wiley: Wiley Online Library; 1996. p. 1841-6.

[10] Li Zhiqiang, Gmitro Arthur F, Bilgin Ali, Altbach Maria I. Fast decomposition of water and lipid using a GRASE technique with the IDEAL algorithm. Magn Reson Med 2007;57(6):1047-57.

[11] Kim Hahnsung, Kim DongHyun, Park Jaeseok. Variable-flip-angle single-slab 3D GRASE imaging with phase-independent image reconstruction. Magn Reson Med 2015;73(3):1041-52.

[12] Kim Hahnsung, Kim Donghyun, Sohn Chulho, Park Jaeseok. Rapid chemical shift encoding with single-acquisition single-slab 3D GRASE. Magn Reson Med 2017;78(5):1852-61.

[13] Pruessmann Klaas P, Weiger Markus, Scheidegger Markus B, Boesiger Peter. SENSE: sensitivity encoding for fast MRI. Magn Reson Med 1999;42(5):952-62.

[14] Griswold Mark A, Jakob Peter M, Heidemann Robin M, Nittka Mathias, Jellus Vladimir, Wang Jianmin, et al. Generalized autocalibrating partially parallel acquisitions (GRAPPA). Magn Reson Med 2002;47(6):1202-10. 
[15] Brau AC, Beatty PJ, Skare S, Bammer R. Efficient computation of autocalibrating parallel imaging reconstruction. Proceedings of the 14th Annual Meeting of ISMRM. 2006. p. 2462.

[16] Bilgic Berkin, Kim Tae Hyung, Liao Congyu, Manhard Mary Kate, Wald Lawrence L, Haldar Justin P, et al. Improving parallel imaging by jointly reconstructing multicontrast data. Magn Reson Med 2018;80(2):619-32.

[17] Breuer Felix A, Blaimer Martin, Mueller Matthias F, Seiberlich Nicole, Heidemann Robin M, Griswold Mark A, et al. Controlled aliasing in volumetric parallel imaging (2D CAIPIRINHA). Magn Reson Med 2006;55(3):549-56.

[18] American College of Radiology (ACR). Phantom test guidance for the ACR MRI Accreditation Program. Reston, Va: ACR; 1998.

[19] Hansen Per Christian. Rank-deficient and discrete ill-posed problems: numerical aspects of linear inversion. Philadelphia: Siam; 1998.

[20] Robson Philip M, Grant Aaron K, Madhuranthakam Ananth J, Lattanzi Riccardo, Sodickson Daniel K, McKenzie Charles A. Comprehensive quantification of signalto-noise ratio and g-factor for image-based and k-space-based parallel imaging reconstructions. Magn Reson Med 2008;60(4):895-907.

[21] Walsh David O, Gmitro Arthur F, Marcellin Michael W. Adaptive reconstruction of phased array MR imagery. Magn Reson Med 2000;43(5):682-90.

[22] Smith Stephen M. Fast robust automated brain extraction. Hum Brain Mapp 2002;17(3):143-55.

[23] Chavhan Govind B, Babyn Paul S, Thomas Bejoy, Shroff Manohar M, Mark Haacke E. Principles, techniques, and applications of T2*-based MR imaging and its special applications. Radiographics 2009;29(5):1433-49. 